\title{
Epidemiologia do óbito fetal em população de baixa renda
}

\author{
Epidemiology of fetal death in a low income population
}

Márcia M. A. de Aquino, José Guilherme Cecatti

\section{RESUM0}

A morte fetal não é uma entidade rara e, em países em desenvolvimento, suas causas mais prevalentes continuam sendo passíveis de controle e/ou tratamento. O objetivo deste estudo foi investigar causas de morte fetal em uma população brasileira. Foi um estudo descritivo realizado no Hospital Maternidade Leonor Mendes de Barros, em São Paulo. Foram estudadas 122 gestantes com diagnóstico de óbito fetal e idade gestacional de vinte semanas ou mais. Os procedimentos estatísticos utilizados foram média e desvio-padrão. As principais causas de morte identificadas foram hipertensão arterial e infecções e em um quarto dos casos a causa não foi determinada. Concluiu-se que uma proporção importante de óbitos era prevenivel e que houve taxa significativa de causas não-identificadas. Os resultados deste estudo poderão ser úteis para orientação de programas de prevenção primária, principalmente quanto à assistência pré-natal.

PALAVRAS-CHAVE: Óbitofetal. Pré-Natal. Complicações da gestação. Infecções. Hipertensão.

\section{Introdução}

A morte fetal tardia, a despeito de todo o avanço tecnológico existente atualmente na área médica, não é uma entidade rara. Ocorre em porcentagem que varia de 0,6 a $1,2 \%$ das gestantes na segunda metade da gestação ${ }^{7}$.

Há divergências entre os autores para delimitação da idade gestacional e de outros parâmetros, como peso e estatura, para definir a morte fetal.

A Organização Mundial de Saúde (OMS) define morte fetal como aquela que ocorre antes da completa expulsão ou extração do produto da concepção do organismo materno, independente da duração da gestação ${ }^{19}$. O National Center for Health Statistics dos Estados Unidos estabeleceu que a expressão morte fetal deve ser empregada a

Hospital Maternidade Leonor Mendes de Barros;

Departamento de Tocoginecologia CAISM/FCM/UNICAMP

Correspondência: José Guilherme Cecatti

Caixa Postal 6030

13083-970 Campinas - SP

Fax: 019-2393687 partir de 20 semanas completas de gestação, definição adotada pela maioria dos autores ${ }^{13}$.

Classifica-se ainda o óbito fetal de acordo com a época do acontecimento, em anteparto e intraparto. A classificação do óbito fetal dessa forma é de grande importância, já que exibem grandes diferenças no que diz respeito à etiologia, complicações maternas e assistência obstétrica ${ }^{20}$.

A despeito da importância evidente da morte fetal como um problema de saúde pública, pouca atenção tem sido dada a esse tema, principalmente quanto à sua epidemiologia, raramente se tem analisado a mortalidade fetal separadamente das mortalidades perinatal e infantil ${ }^{14}$.

Para poder reduzir a incidência da morte fetal no período anteparto, é indispensável ter conhecimento sobre sua etiologia. No entanto, 40 a 50\% dos casos são ditos de causa não definida, mesmo em países com maiores recursos diagnósticos ${ }^{8,9,17}$.

Quando conhecida, as causas determinadas de morte fetal no período anteparto são divididas em maternas e feto-anexiais. Entre as causas maternas, destacam-se condicões patológicas tais 
como síndromes hipertensivas, endocrinopatias, anemias em geral, infecções, isoimunização Rh e a presença no soro materno de anticorpos antifosfolípides. Entre essas, as síndromes hipertensivas contituem a principal causa conhecida de morte fetal $^{2,9,16}$.

Quanto às infecções, dentre as bacterianas destacam-se a sífilis e a corioamnionite grave. Em estudo realizado em Ribeirão Preto, no período de 1978 a 1984, observou-se uma taxa de mortalidade fetal por sífilis de 4,5 por 1000 nascimentos e uma prevalência de sífilis entre as mulheres em trabalho de parto de $2,1 \%{ }^{6}$.

A corioamnionite é decorrente de uma infecção ascendente através do canal de parto, cuja etiologia nem sempre pode ser reconhecida. Em um estudo caso-controle realizado na África, verificou-se que a corioamnionite ocorreu com pequena 2,6 vezes maior em mulheres com natimortos do que em mulheres com recém-nascido vivo ${ }^{11}$. Já entre as infecções parasitárias incluemse a Doença de Chagas, a toxoplasmose e a malária. $\mathrm{E}$, entre as virais, a citomegalovirose e a infecção pelo herpes vírus tipo $\mathrm{II}^{3}$.

$\mathrm{O}$ óbito intra-uterino na mulher diabética pode ser determinado pelas alterações metabólicas, principalmente a hiperglicemia, a hipoglicemia e a cetoacidose, que ocorrem na presença de um deficiente controle do diabetes e, também, por anomalias congênitas e hipóxia. No estudo de Pereira ${ }^{12}$ observou-se uma prevalência de $4,4 \%$ de óbitos fetais entre gestantes diabéticas, dos quais um quarto apresentava malformação congênita.

A desnutrição, o uso de quimioterápicos, antineoplásicos, anticoagulantes orais e drogas ilícitas pela gestante, além das intoxicações por metais pesados e as alterações uterinas são causas menos freqüentes de morte fetal ${ }^{5,9}$.

As causas fetais mais importantes são as malformações congênitas e as anormalidades cromossômicas que, para serem diagnosticadas, dependem de protocolos que incluam a necrópsia do recém-nascido e o estudo citogenético, ficando muito prejudicados quando o decesso não é recente ${ }^{15}$

Quanto às causas anexiais, destacam-se as hemorragias do terceiro trimestre da gestação, as funiculopatias e também a transfusão na prenhez gemelar monozigótica ${ }^{9,10}$.

De publicações na literatura médica, sabese que as causas associadas ao óbito fetal são muitas, o que dificulta o diagnóstico de sua etiologia. Além disso, é necessário estudar a importância relativa das diferentes causas em diferentes populações. Nesse sentido, o propósito deste estudo foi investigar a morte fetal, a fim de verificar quais as causas mais prevalentes de óbito fetal anteparto em uma população brasileira de baixa renda.

\section{Material e métodos}

Trata-se de estudo descritivo no qual foram analisadas 122 mulheres com diagnóstico de óbito fetal atendidas no período de agosto de 1996 a maio de 1997. A amostra de casos foi obtida segundo a ordem de internação no Setor de Admissão da instituição que presta atendimento à população de baixa renda da zona leste da cidade de São Paulo. Foram incluídas gestantes com diagnóstico de óbito fetal, ocorrido antes do início do trabalho de parto, com idade gestacional igual ou superior a vinte semanas. Este estudo foi desenvolvido com mulheres que participaram voluntariamente, após terem sido esclarecidas sobre o estudo e assinado um termo de consentimento pós-informação. O projeto de pesquisa foi previamente analisado e aprovado pelo Comitê de Ética em Pesquisa da Instituição.

Excluíram-se as gestantes com óbito fetal que tenham ocorrido após algum procedimento obstétrico invasivo (amniocentese, cordocentese, biópsia de vilo corial, etc) que tenha ocasionado a morte do feto por hemorragia.

Para coleta dos dados foram utilizados um questionário estruturado e um formulário onde constavam os resultados dos exames laboratoriais, ultra-sonográfico, dados clínicos e os resultados dos exames histológicos das placentas e laudo das necrópsias dos recém-nascidos. Esses instrumentos foram revisados quanto à qualidade e legitimidade das informações, e os dados codificados e armazenados em arquivo de computador, após testes de consistência.

Quanto à análise desses dados, procedeu-se à análise descritiva dos dados referentes às prováveis causas de óbito fetal e às características sociodemográficas e gestacionais das mulheres. Utilizou-se o pacote estatístico EPI INFO 6.02.

\section{Resultados}

De $23 \%$ das pacientes que tinham antecedentes de uma ou mais cesáreas anteriores. O parto foi induzido com misoprostol em 37,3\% dos casos, com ocitocina em $20,3 \%$, com ambos em $16,1 \%$ e iniciou espontaneamente em $26,3 \%$ dos casos. A via de parto foi vaginal em $90,6 \%$ das pacientes e cesárea nas demais. A indicação de cesárea deu-se por interatividade na grande maioria dos casos, e apenas um caso por falha de indução.

As mulheres estudadas eram jovens, com média de idade em torno de 27 anos, sendo a maioria branca e em união conjugal. As mulheres apresentaram, como antecedente, média de 2 
gestações anteriores, compareceram a 4 consultas de pré-natal.

A medida dos pesos fetais foi de $1500 \mathrm{~g}$. A renda familiar em salários-mínimos foi em torno de 6 , e a maior parte das mulheres não realizava trabalho remunerado. Em relação à escolaridade, chama a atenção que 63\% das mulheres tinham cursado somente até o ginásio incompletamente (Tabelas 1 e 2).

Tabela 1 - Idade, renda familiar e características da gestações e dos fetos; desvio padrão e faixa de variação.

\begin{tabular}{|c|c|c|c|c|c|}
\hline & $\mathrm{N}$ & Média & DP & Min & Max \\
\hline Idade (anos) & 122 & 26,9 & 7,5 & 12 & 48 \\
\hline Renda familiar $(\mathrm{SM})^{\mathrm{S}}$ & $108^{*}$ & 5,6 & 2,8 & 1,0 & 14,0 \\
\hline Idade gestacional (sem.) & 122 & 28,4 & 6,0 & 20 & 41 \\
\hline$N^{o}$ de gestações & 122 & 2,9 & 2,3 & 01 & 12 \\
\hline $\mathrm{N}^{\circ}$ de consultas pré-natal & $117^{\#}$ & 3,9 & 2,4 & 00 & 10 \\
\hline Peso dos natimortos (g) & $120^{\S}$ & 1467,4 & 1087,3 & 150 & 4870 \\
\hline
\end{tabular}

* Faltam informações de 14 casos

* Faltam informações de 5 casos

$\S$ Faltam informações de 2 casos

s Salário mínimos.

Tabela 2 - Distribuição das gestantes segundo algumas características sociodemográficas

\begin{tabular}{|c|c|}
\hline Escolaridade (série) & $\%$ \\
\hline Primário incompleto & 15,7 \\
\hline Primário completo & 13,0 \\
\hline Ginásio incompleto & 33,9 \\
\hline Ginásio completo & 15,7 \\
\hline Colegial incompleto & 7,8 \\
\hline Colegial completo ou superior incompleto ou completo & 13,9 \\
\hline$\left(n^{*}\right)$ & $(115)$ \\
\hline \multicolumn{2}{|l|}{ Trabalho } \\
\hline $\operatorname{Sim}$ & 33,6 \\
\hline Não & 66,4 \\
\hline (n) & $(122)$ \\
\hline \multicolumn{2}{|l|}{ Estado marital } \\
\hline Solteira & 16,4 \\
\hline Casada/amasiada/vive junto & 82,0 \\
\hline Separada/divorciada/viúva & 1,6 \\
\hline (n) & $(122)$ \\
\hline \multicolumn{2}{|l|}{ Cor } \\
\hline Branca & 59,8 \\
\hline Parda & 32,0 \\
\hline Negra & 8,2 \\
\hline (n) & $(122)$ \\
\hline
\end{tabular}

* Faltam informações de 7 casos.

Em relação ao peso fetal, observou-se que cerca de $2 / 3$ dos $\mathrm{RN}$ apresentaram peso inferior a 1500 g. Apenas cerca de $15 \%$ dos casos tiveram causa certa e, aproximadamente, na metade deles pode ser determinada a causa provável da morte fetal. De todas as causas isoladas, a mais prevalente foi a hipertensão arterial $(20,5 \%)$. Observaram-se 10 casos de descolamento prematuro de placenta (DPP), todos com hipertensão arterial associada. Somados aos 25 casos em que a causa provável foi hipertensão, encontrou-se que em quase $30 \%$ dos casos de morte fetal a hipertensão arterial esteve presente como fator etiológico. As várias causas infecciosas foram responsáveis por $13,2 \%$ das mortes e, entre elas, a sífilis foi diagnosticada em cinco casos $(4,2 \%)$. Em quase um quarto dos casos não se pode definir a causa do óbito (Tabela 3).

Tabela 3 - Distribuição percentual dos casos, segundo as causas de óbito fetal.

\begin{tabular}{|c|c|c|c|}
\hline Causas & $\mathbf{n}$ & & $\%$ \\
\hline Indeterminadas & 30 & 24,6 & \\
\hline Possíveis & 16 & 13,1 & \\
\hline AIDS* & 03 & & 2,5 \\
\hline Placenta prévia centro-total & 02 & & 1,6 \\
\hline Infecção intra-uterina inespecífica & 07 & & 5,7 \\
\hline Uso de cocaína & 03 & & 2,5 \\
\hline Epilepsia & 01 & & 0,8 \\
\hline Prováveis & 58 & 47,5 & \\
\hline Hipertensão & 25 & & 20,5 \\
\hline Diabetes & 07 & & 5,7 \\
\hline Alterações do cordão umbilical ${ }^{\#}$ & 04 & & 3,3 \\
\hline Malformações fetais $\S$ & 10 & & 8,2 \\
\hline Insuficiência placentária & 11 & & 9,0 \\
\hline Hidropisia fetal & 01 & & 0,8 \\
\hline Certas & 18 & 14,8 & \\
\hline DPP & 10 & & 8,2 \\
\hline Toxoplasmose & 01 & & 0,8 \\
\hline Cardiopatia fetal $\dagger$ & 02 & & 1,6 \\
\hline Sífilis & 05 & & 4,2 \\
\hline (n) & (122) & & (100) \\
\hline
\end{tabular}

* Síndrome da Imunodeficiêcia Adquirida. \# Nó verdadeiro de cordão, circular cervical apertada, artéria umbilical única, 3 circulares + nó verdadeiro. § 6 casos de anomalias do Sistema Nervoso Central, 1 caso de anomalia do Sistema Esquelético e 3 casos de malformações múltiplas. † Tetrologia de Fallot e defeito atrioventricular septal forma total.

\section{Discussão}

O presente estudo foi motivado pelo interesse no conhecimento das causas de óbito fetal na população brasileira, em especial na de baixa renda, onde sua ocorrência é maior. Os resultados obtidos mostram que as principais causas identificadas de morte fetal foram a hipertensão arterial e as infecções. 
Entretanto, em apenas uma de cada sete mortes foi determinada a causa com certeza e em uma de cada quatro mortes a causa permaneceu indefinida, sem a identificação de qualquer fator etiológico suspeito de causar o óbito intra-uterino. $\mathrm{O}$ indice de causas indeterminadas encontrado neste estudo está dentro dos amplos limites relatados na literatura médica que refere cifras variando de 12 a $52 \%$, na maioria dos trabalhos ${ }^{1,17,18}$.

Ter encontrado a hipertensão arterial como a principal causa de morte fetal nessa população não surpreende, já que outros estudos realizados em países em desenvolvimento identificaram essa mesma causa ${ }^{2,16}$. Este estudo mostrou uma taxa em torno de $6 \%$ de óbitos fetais atribuíves às infecções intra-uterinas inespecíficas, índice próximo ao encontrado por Copper e cols. ${ }^{4}$ que foi de 9,4\%. Entre essas causas infecciosas, a toxoplasmose e a sífilis foram classificadas como causa certa do óbito porque se confirmou o diagnóstico de infecção pela sorologia e havia vilosite no exame histológico da placenta. No único caso de toxoplasmose observou-se nesse exame a presença de vilosite necrotizante e granuloma de células gigantes com a identificação do Toxoplasma gondii.

É razoável assumir que as infecções intrauterinas inespecíficas tenham sido a causa da morte porque, além do diagnóstico clínico, confirmou-se a presença da infecção através do exame histológico da placenta, observou-se presença de vasculite e corioamnionite, o que sugere que o feto estava vivo quando a infecção ocorreu $^{11}$.

A prevalência de insuficiência placentária como causa de morte foi de 9\% neste estudo. Incluíram-se nesse diagnóstico os casos confirmados pela presença de infartos placentários no exame histológico da placenta, critério também utilizado por Ahlenius e cols. ${ }^{1}$ que atribuíram a essa etiologia $8 \%$ de sua casuística. Até onde chega o nosso conhecimento, esse é o primeiro estudo brasileiro avaliando causas de morte fetal contando com o exame histológico de todas as placentas da população de mulheres.

As principais causas de morte encontradas neste estudo foram semelhantes àquelas que aparecem com maior freqüência na literatura médica. Porém, as diferenças na porcentagem das casuísticas em relação a outros estudos poderiam ser explicadas pelas características das populações estudadas, no que diz respeito ao nível socioeconômico, qualidade da assistência pré-natal e nível de saúde, o que acaba alterando a ordem das principais causas de morte fetal.

Este estudo mostrou que, em nosso país, a causa mais prevalente de morte fetal continua sendo a hipertensão arterial, o que sugere que o diagnóstico precoce da hipertensão durante a gestação e a intervenção médica adequada poderiam ajudar a reduzir o risco de natimortalidade associada a essa causa.

Para a detecção precoce de condições patológicas na gestação é necessário que se disponha de um adequado programa de assistência pré-natal, oferecido em quantidade e qualidade suficientes para a população, sobretudo a de baixa renda, que dele necessita.

É possível dizer que este estudo deu uma contribuição aos conhecimentos sobre causas de morte fetal tardia no período anteparto em uma população brasileira de baixa renda. Mostrou que, no Brasil, as causas mais prevalentes continuam sendo a hipertensão arterial e as infecções, que são processos passíveis de controle e/ou tratamento: a identificação e o tratamento precoces de sífilis, diabetes e hipertensão poderão reduzir os índices de morte fetal anteparto na população brasileira, dependendo de ações de saúde e assistência médica adequada.

Os resultados deste estudo poderão ser úteis para a orientação de programas de prevenção primária, principalmente quanto à assistência prénatal, fornecendo informações para a implantação de possiveis programas que enfatizem a necessidade do seguimento e tratamento de condições patológicas que podem ocasionar a morte fetal. Poderá também orientar os profissionais da saúde para estarem atentos em relação às mulheres que apresentaram morte fetal em sua última gestação, esclarecendoas em seu retorno de puerpério, tratando-as quando necessário e sugerindo os métodos anticoncepcionais adequados.

\section{SUMMARY}

Fetal death may not be considered an unusual event and, in developing countries, the most prevalent causes could be possibly controlled and/or treated. The purpose of the present study was to investigate causes of fetal death in a Brazilian population. This is a descriptive study performed at the Hospital Maternidade Leonor Mendes de Barros in São Paulo. The study subjects were 122 pregnant women with diagnosis of fetal death and gestation age of 20 or more weeks. The statistical procedures used were means and standard deviation. The main causes of the fetal death were hypertensive disorders and infections and, for a quarter of the cases, they were not identified at all. It is concluded that an important percentage of fetal deaths would have been prevented and that there was a significant number of unidentified causes. Results of the present study might be useful to orientate a primary prevention health program, specially concerning antenatal care.

KEY WORDS: Stillbirth. Prenatal care. Complications of pregnancy. Infections. Hypertension. 


\section{Referências bibliográficas}

1. Ahlenius I, Floberg J, Thomassen P. Sixty-six cases of intrauterine fetal death. A prospective study with an extensive test protocol. Acta Obstet Gynecol Scand 1995; 74:109-17.

2. Axemo P, Liljestrand J, Bergstrom S, Gebre-Medhin M. A etiology of late fetal death in Maputo. Gynecol Obstet Invest 1995; 39:103-9.

3. Benirschke K \& Robb J.A. Infectious causes of fetal death. Clin Obstet Gynecol 1987; 30:284-93.

4. Copper RL, Goldenberg RL, DuBard MB, Davis RO. Risk factors for fetal death in white, black and hispanic women. Obstet Gynecol 1994; 84: 490-495.

5. Della Nina M, Peixoto S. Óbito intra-uterino. In: Peixoto S. Pré-natal. São Paulo, Manole, 1981 p.773-87.

6. Duarte G, Cunha SP, Yamada RT, Bailão L A, Philbert PMP. Sífilis e gravidez: ainda um problema. Rev Bras Ginecol Obstet 1987; 97:75-78.

7. Kubli F. Muerte fetal intrauterina en la segunda mitad del embarazo. In: Kaser O. Ginecologia y Obstetricia. Barcelona, Salvat, 1974 p. 115.

8. Lau TK \& Li C Y. A perinatal audit of stillbirths in a teaching hospital in Hong Kong. Aust. N. Z. J Obstet Gynaecol 1994; 34: 416-21.

9. Mariani Neto C. Óbito fetal. In: Neme B. Obstetrícia Básica. São Paulo, Sarvier, 1994 p. 382-6.

10. Modotte WP, Dias R, Lima CDC. Síndrome de transfusão feto-fetal. Rev Bras Ginecol Obstet 1997; 19: 223-6.

11. Moyo SR, Hagestrand I, Nystrom L, Tswana SA, Blomberg J, Bergstrom S, Ljungh A. Stillbirths and intrauterine infection, histologic chorio- amnionitis and microbiological findings. Int $\mathbf{J}$ Gynaecol Obstet 1996; 54:115-23.

12.Pereira BG. Avaliação de um protocolo de assistência à grávida diabética. Campinas, 1992 [Dissertação - Mestrado - Universidade Estadual de Campinas].

13.Petitti DB. The epidemiology of fetal death. Clin Obstet Gynecol 1987; 30:253-8.

14. Raymond EG, Cnattingius S, Kiely JL. Effects of maternal age, parity and smoking on the risk of stillbirth. Br J Obstet Gynaecol 1994; 101: 301-6.

15.Richard MP, Reiser CA, Lebovitz RM, Kirkpatrick SJ. Wisconsin Stillbirth Service Program: I. Establishment and assessment of a community - based program for etiologic investigation of intrauterine deaths. Am J Med Genet 1994; 50:116-34.

16.Urbanetz AA, Galloro Filho F, Kawai R, Dondoni R, Sampaio G. Óbito fetal intraútero: Análise de 176 casos. In: XV Congresso Brasileiro de Ginecologia e Obstetrícia, São Paulo, 1989. Anais. São Paulo, 1989 p. 45-56.

17.Walles B, Tyden T, Herbst A, Ljungblad U, Rydhstrom H. Maternal health care program and markers for late fetal death. Acta Obstet Gynecol Scand 1994; 73: 773-8.

18.Were EO. Stillbirths at Eldoret District Hospital: a retrospective study. East Afr Med J 1994; 71: 607-10.

19.World Health Organization. Recommended definitions, terminology and format for statistical tables related to the perinatal period and use of a new certificate for cause of perinatal deaths. WHO/ FIGO. Acta Obstet Gynecol Scand 1977; 56: 247-53.

20.Zlatinik FJ. Management of fetal death. Clin Obstet Gynecol 1986; 29: 220-229. 